\title{
Modeling soft granular materials
}

\author{
Saeid Nezamabadi $^{1}$. Thanh Hai Nguyen ${ }^{1,3}$ - Jean-Yves Delenne ${ }^{2}$. \\ Farhang Radjai ${ }^{1,4}$
}

\begin{abstract}
Soft-grain materials such as clays and other colloidal pastes share the common feature of being composed of grains that can undergo large deformations without rupture. For the simulation of such materials, we present two alternative methods: (1) an implicit formulation of the material point method (MPM), in which each grain is discretized as a collection of material points, and (2) the bonded particle model (BPM), in which each soft grain is modeled as an aggregate of rigid particles using the contact dynamics method. In the MPM, a linear elastic behavior is used for the grains. In order to allow the aggregates in the BPM to deform without breaking, we use long-range center-to-center attraction forces between the primary particles belonging to each grain together with steric repulsion at their contact points. We show that these interactions lead to a plastic behavior of the grains. Using both methods, we analyze the uniaxial compaction of $2 \mathrm{D}$ soft granular packings. This process is nonlinear and involves both grain rearrangements and large deformations. High packing fractions beyond the jamming state are reached as a result of grain shape change for both
\end{abstract}

$\triangle$ Saeid Nezamabadi

saeid.nezamabadi@umontpellier.fr

1 Laboratoire de Mécanique et Génie Civil (LMGC), Université de Montpellier, CNRS, Montpellier, France

2 IATE, UMR1208 INRA - CIRAD - Université de Montpellier - SupAgro, 34060 Montpellier, France

3 Water Resources Engineering Department, University of Science and Technology, 54 Nguyen Luong Bang St, LienChieu Dist, Da Nang, Vietnam

$4<$ MSE $>$ ${ }^{2}$, UMI 3466 CNRS-MIT, CEE, Massachusetts Institute of Technology, 77 Massachusetts Avenue, Cambridge, MA 02139, USA methods. We discuss the stress-strain and volume change behavior as well as the evolution of the connectivity of the grains. Similar textures are observed at large deformations although the BPM requires higher stress than the MPM to reach the same level of packing fraction.

Keywords Granular materials $\cdot$ Soft matter $\cdot$ Material point method - Contact dynamics - Discrete element method . Elasto-plastic behavior

\section{Introduction}

Many materials are composed of a disordered network of soft grains. Most food products, metal powders, colloidal suspensions and clays are soft-grain systems [17, 18,20,23]. The interplay between disorder and large deformations of soft grains leads to novel physical and mechanical properties that are out of reach within hard-grain models. High deformability of soft grains under low confining pressure allows for packing fractions above the random close packing of hard grains (unless the grains break). Therefore a combination of grain rearrangements and grain shape change controls the rheological properties such as compressibility and shear strength, as well as the microstructure of the material. The latter can take a wide variety of forms depending on the grain properties (compressibility, plasticity...), interfacial forces (friction, adhesion, repulsion...) between grains and the properties of the suspending fluid. The constitutive grains of soft materials are mostly macromolecular or aggregates of a size ranging from $1 \mathrm{~nm}$ to $1 \mathrm{~mm}$. Based on both their composition and architecture, they can be divided into four groups [7]: colloidal-like grains, network particles, polymercolloid systems and surfactant particles. Although all these 
particles are soft and elastic, the origin of their elasticity and deformability depends on their composition and structure.

Efficient numerical strategies based on discrete modeling have been developed for the simulation of grain assemblies by assuming that the grains are hard or weakly deformable. This approach is now a mature and well-established approach employed in powder technology, soil mechanics and geology. The discrete element method (DEM) and contact dynamics (CD) method are the most common discrete methods for hard grains [32]. In order to allow each grain to deform according to a continuum constitutive behavior, and also to describe the contact interactions, it is necessary to treat both frictional contacts and grain deformations. A promising framework is provided by meshless models that have already been applied to problems of solid mechanics involving large deformations. One of these numerical models is now mostly known as material point method (MPM) [5]. This is a mixed method based on a combination of the Eulerian and Lagrangian descriptions of the material. It can be also considered as a finite element method with moving integration points (material points). The Lagrangian description is provided by discretizing each body as a collection of material points, and the Eulerian description is based on a background computational mesh. The material points are assigned fixed masses during computation so that the conservation of mass is satisfied implicitly. The information carried by the material points is projected onto the background mesh, where the equations of motion are solved. The mesh solution is then used to update the material points. Hence, the momentum changes are interpolated from the grid to the material points, so that the total momentum is conserved. The MPM combines the advantages of Eulerian and Lagrangian methods by avoiding the distortion of Lagrangian mesh and tracking the boundaries of bodies. This method has already been applied to granular materials with a few grains [5, 10,28].

An alternative approach is the bonded particle model (BPM), which consists in modeling each grain as an aggregate composed of hard particles with cohesive interactions such that a grain can deform as a result of the relative motions of the particles while staying together as a solid grain. This "granular discretization" of the grains is a simple approach as it allows for straightforward application of the DEM for the simulation of deformable grains. It has been applied for the simulation of crushable grains by introducing a breaking threshold between particles [2,6,19,27,29,45,48]. But it has never been applied for soft deformable grains.

In this paper, we introduce an implicit MPM and a BPM algorithm for the simulation of soft grains. We apply both methods to investigate the compaction of a packing of soft grains. The soft-grain packings may potentially reach high packing fractions by grain shape change and still deform elastically or plastically. The compaction, volume change behavior under shearing and the properties of the resulting textures above the random close packing state have remained largely unexplored because of the lack of proper numerical and experimental tools in the past. We show that both the MPM and the BPM allow for compaction beyond the random close packing fraction. We analyze the evolution of the packing and the effect of grain shape change. As we shall see, both methods lead to similar textures but the material behavior of the grains affects the stress level and its evolution during compaction.

In the following, we first briefly introduce the numerical approaches. Then, we focus in Sect. 3 on the behavior of a single grain subjected to axial strain. In Sect. 4, we analyze the compaction process of a packing of soft circular grains. We conclude with a brief summary and perspectives of this work.

\section{Numerical methods}

Soft grains have mainly been considered in numerical simulations only in the Hertz limit where small deformations (below 5\%) of spherical grains at their contact points are taken into account on an analytical basis. The power-law force-displacement relation is used in the DEM as a force law while keeping the reference spherical shape as the reference geometry for the calculation of local strain variables $[1,32]$. In other words, the grains are treated as rigid elements but interacting through soft contacts. Large deformations of soft grains ("ultra-soft" grains) can only be achieved by introducing the internal degrees of freedom of the grains either within continuum mechanics or by representing the grains as an agglomerate of interacting discrete particles. We describe below two algorithms based on these two different representations that will be applied to the compaction process of a collection of soft grains.

\subsection{Bonded particle model (BPM)}

We use the BPM as a generic term for models in which each grain is an aggregate of primary rigid particles with cohesive interactions. The rigid particles in an aggregate can move and rearrange according to the external forces acting on its boundaries by other aggregates. When the interactions between primary particles are governed by an irreversible cohesive law, i.e. when contact adhesion can be lost irreversibly, this model can be used to model crushable grains $[21,29,38,47]$. In this case, the grains undergo irreversible rupture and may turn into several fragments when the failure threshold is reached at several contact points between the particles. To allow for large deformations of the grains without irreversible loss of interactions between primary particles, it is therefore necessary to replace contact adhesion (which is always short-ranged) by a long-range center-to-center attrac- 


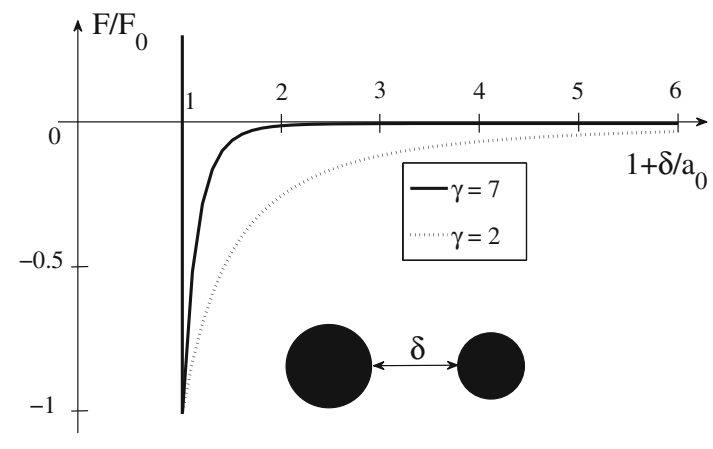

Fig. 1 Lennard-Jones attraction force law between primary particles for two values of the exponent $\gamma$; see Eq. (1)

tion force, as in colloidal systems, together with a short-range repulsion force [15].

Let us consider two rigid disks of radii $a_{1}$ and $a_{2}$. For the attraction force acting between primary particles, we choose the attraction part of a generalized Lennard-Jones (L-J) force law $[14,15]$ :

$$
F_{a}=-F_{0}\left(1+\frac{\delta}{a_{0}}\right)^{-\gamma}
$$

where $a_{0}=a_{1}+a_{2}$ and $F_{0}$ is the maximum absolute value of attraction force for the inter-particle distance $\delta=0$. For the original L-J potential, we have $\gamma=7$, but the same attraction law may be generalized to arbitrary values of $\gamma$ to represent the potential of the mean force in complex materials such as cement and clay [14].

For the repulsive part of the interaction, we assume that the particles are perfectly rigid so that the repulsion force $F_{r}$ is activated only when the two particles touch, i.e. for a gap $\delta=0$. Hence, the interaction force $F=F_{a}+F_{r}$ as a function of $\delta$ consists of only attraction at arbitrary distance $\delta>0$ and takes a value $\in\left[-F_{0},+\infty\right]$ at contact $(\delta=0)$. The graph of this interaction law is displayed in Fig. 1 for $\gamma=2$ and $\gamma=7$. For an efficient implementation of this law, a cutoff is necessary on the inter-particle distance. However, for $\gamma=7$ the attraction force is negligibly small beyond $\delta \simeq a_{0}$, in which case only the first and second neighbors of each particle are involved in the interactions with other particles. This distance is short but long enough to keep the particles belonging to a grain together during large deformations.

The repulsive part can be modeled by introducing either a power law as in the original L-J potential or a simple spring as usual in DEM simulations. But we use a contact dynamics approach, in which unilateral contacts are treated in the framework of an implicit time-stepping algorithm $[26,34]$. This approach has the advantage of eluding the use of stiff repulsive potentials and hence the numerical treatment of small elastic strains at the contact points. The $\mathrm{CD}$ is a general method for the treatment of frictional

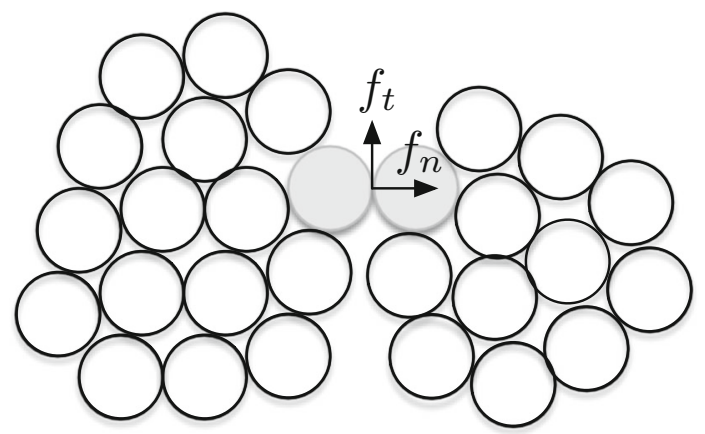

Fig. 2 A frictional contact between two primary particles belonging to two different grains with normal and tangential components $f_{n}$ and $f_{t}$ of the contact force

unilateral contacts without introducing specific force laws. The rigid-body equations of motion are integrated by taking into account the kinematic constraints resulting from contact interactions. These interactions are characterized by three parameters: the coefficient of friction and the coefficients of normal and tangential restitution that control the rate of dissipation. The implicit time-stepping scheme makes the method unconditionally stable. For this reason, the time step can be larger than that in molecular dynamics method. An iterative algorithm similar to nonlinear Gauss-Seidel method is used to determine the contact forces and particle velocities simultaneously at all eligible contacts between particles. Detailed descriptions of the foundations and algorithmic features of the $\mathrm{CD}$ method can be found in the literature $[16,24,34]$. This method has been extensively used for the simulation of granular materials with rigid grains in $2 \mathrm{D}$ and $3 \mathrm{D}[3,4,8,11,25,29,31,33,35-37,41,42]$.

Since we are interested here in soft deformable grains, we set the friction coefficient between the primary particles to zero. As a result, the particle rotations inside a grain are immaterial and no energy dissipation occurs inside the grains by friction. However, the restitution coefficients are set to zero, thus allowing the energy be dissipated by collisions between the particles. The deformation of each grain involves both a small reversible part due to the action of attraction forces and a plastic part arising from rearrangements of primary particles. It is important to remark that, in the absence of friction between primary particles, the volume of each grain is nearly constant since the rearrangements occur without dilatancy [30].

Although no friction is considered between primary particles inside each grain, we may introduce frictional contacts between the grains. These contacts occur between two primary particles belonging to the two grains, as illustrated in Fig. 2. Hence, we distinguish two contact types between primary particles: (1) for two touching primary particles belonging to the same grain, the contact is always frictionless but cohesive; (2) for two touching primary particles belong- 
ing to two different grains, the contact is always non-cohesive (no attraction force) but can be frictional. We will consider below both frictional and frictionless contacts between grains.

\subsection{Material point method (MPM)}

We briefly present here the MPM, as a meshless finite element method for the simulation of soft grains, coupled with the CD method for the treatment of frictional contacts between grains. More details of the method can be found in [28].

Let us consider a domain $\Omega$ in $\mathbb{R}^{D}, D$ being its dimension, describing a continuum body with an external boundary $\partial \Omega$. The conservation of mass is governed by the following continuity equation:

$$
\frac{\partial \rho(\mathbf{x}, t)}{\partial t}+\nabla \cdot(\rho(\mathbf{x}, t) \mathbf{v}(\mathbf{x}, t))=0 \quad \text { in } \Omega
$$

where $\rho(\mathbf{x}, t)$ is the material density and $\mathbf{v}(\mathbf{x}, t)$ denotes the velocity at position $\mathbf{x}$ and time $t$. In the same way, the conservation of linear momentum is given by

$$
\nabla \cdot \sigma(\mathbf{x}, t)+\mathbf{b}(\mathbf{x}, t)=\rho(\mathbf{x}, t) \mathbf{a}(\mathbf{x}, t) \text { in } \Omega,
$$

where $\sigma(\mathbf{x}, t)$ is the Cauchy stress tensor, $\mathbf{b}(\mathbf{x}, t)$ represents the body force and $\mathbf{a}(\mathbf{x}, t)$ denotes the acceleration at position $\mathbf{x}$ and time $t$. The continuity Eq. (2) and momentum Eq. (3) must be supplemented with a constitutive relationship which is assumed here to be linear, homogeneous, isotropic and elastic:

$\sigma(\mathbf{x}, t)=\mathbb{C}: \boldsymbol{\epsilon}(\mathbf{x}, t)$,

where $\mathbb{C}$ is the fourth-order elastic tensor and $\epsilon$ is the strain tensor $\left(\boldsymbol{\epsilon}=\frac{1}{2}\left(\nabla \mathbf{u}+\nabla \mathbf{u}^{T}\right), \mathbf{u}\right.$ being the displacement field $)$. Other constitutive laws (including inelastic behaviors such as plastic deformations) may be used in this context.

In the framework of the MPM, the simulation domain is divided into $N_{p}$ infinitesimal mass elements. These elements are represented by material points of constant mass. Hence, since the material point mass is considered to be fixed, the mass conservation relation (2) is self-satisfied. Furthermore, the material points serve as integration points to compute the FEM integrals. The MPM then discretizes the variables (stress, strain, density, velocity, acceleration...) through a Dirac delta function by considering a fixed material point mass. Hence, for example, the density may be discretized for an element in the form:

$$
\rho(\mathbf{x}, t)=\sum_{p=1}^{N_{p}} \rho_{p} \delta\left(\mathbf{x}-\mathbf{X}_{p}(t)\right)
$$

where $\rho_{p}$ and $\mathbf{X}_{p}(t)$ refer to material point density and position, respectively, and $\delta$ is the Dirac delta function. The material point density is equal to: $\rho_{p}=m_{p} / V_{p}$ with material point mass $m_{p}$ and material point volume $V_{p}$.

In the same way, the displacement $\mathbf{u}_{p}$ and the strain $\boldsymbol{\epsilon}_{p}$ at material point $\mathbf{X}_{\mathbf{p}}$ are expressed as a function of nodal displacement $\mathbf{u}_{\text {node }}$ as:

$\mathbf{u}_{p}=\mathbf{N}_{p} \mathbf{u}_{\text {node }}$ and $\boldsymbol{\epsilon}_{p}=\mathbf{G}_{p} \mathbf{u}_{\text {node }}$,

where $\mathbf{N}_{p}$ denotes interpolation matrix or shape function matrix at $\mathbf{X}_{\mathbf{p}}$, and $\mathbf{G}_{p}$ is the gradient of the shape function $\mathbf{N}_{p}$. Therefore, in the presence of contact interactions between bodies, the weak form of the equation of motion (3) in a discretized form can be written as

$\mathbf{M} \mathbf{a}_{\text {node }}(t)=\mathbf{F}^{\text {int }}(t)+\mathbf{F}^{\text {ext }}(t)+\mathbf{F}^{\mathrm{C}}(t)$,

where $\mathbf{a}_{\text {node }}$ is the nodal acceleration, $\mathbf{F}^{\mathrm{C}}$ denotes the contact force (see below) and $\mathbf{F}^{\text {ext }}$ represents the external applied force. $\mathbf{M}$ is the lumped mass matrix:

$\mathbf{M}=\sum_{p=1}^{N_{p}} m_{p} \mathbf{N}_{p}$

and $\mathbf{F}^{\text {int }}$ gives the internal force vector resulting from the stress divergence:

$\mathbf{F}^{\mathrm{int}}=-\sum_{p=1}^{N_{p}} \mathbf{G}_{p} \boldsymbol{\sigma}_{p} V_{p}$

Since there are generally more material points than grid nodes, a weighted squares approach is used to determine nodal velocities $\mathbf{v}_{\text {node }}$ from the material point velocities $\mathbf{v}_{p}$. Hence, the nodal velocities are obtained by solving the following relation:

$\mathbf{P}_{\text {node }}=\mathbf{M} \mathbf{v}_{\text {node }}=\sum_{p=1}^{N_{p}} m_{p} \mathbf{N}_{p} \mathbf{v}_{p}$

where $\mathbf{P}_{\text {node }}$ is the nodal momentum.

In order to solve the problem Eq. (7), we consider an implicit MPM approach proposed by Guilkey and Weiss [12]. The nodal solutions are projected onto the material points, allowing for updating the information carried by these points.

In dealing with a packing of deformable grains, the contact forces $\mathbf{F}^{\mathrm{C}}$ between grains need to be calculated by means of a contact algorithm accounting for the condition of noninterpenetration of matter as well as the Coulomb friction law. We used the CD method, which we adapted to the MPM algorithm. The coupling of MPM and CD has been explained 


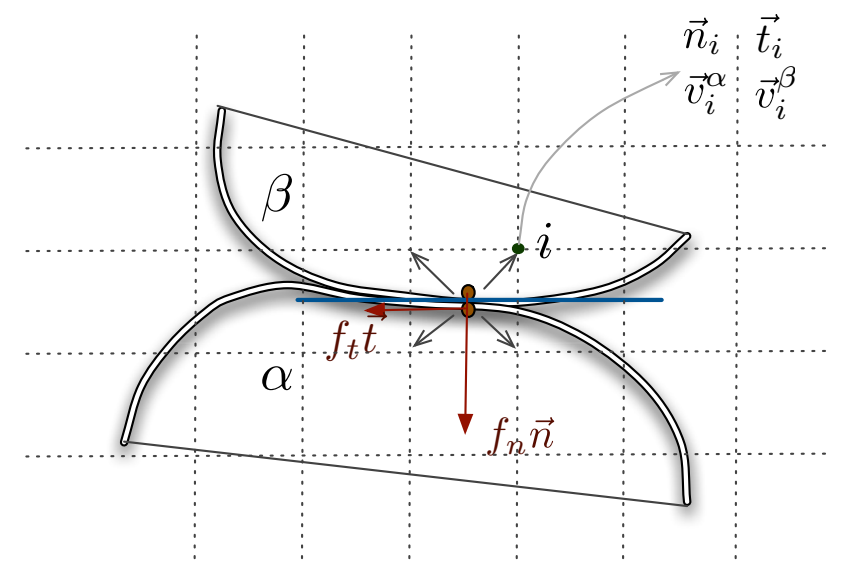

Fig. 3 Multi-mesh contact algorithm scheme in MPM; see text

in detail in a previous paper [28], but, will be described briefly here.

Let us consider two deformable grains ( $\alpha$ and $\beta$ ); see Fig. 3. In the context of multi-mesh algorithm, each grain maps in its proper background mesh. A contact point at the interface between the two grains may be treated by introducing a common background mesh with the same type of grids for the transfer of nodal quantities from proper meshes to the common mesh. The contact points between the grains $\alpha$ and $\beta$ are treated at the neighboring nodes belonging to the common background mesh. Their nodal values involve contributions from the two grains. At a potential contact node $i$, a normal unit vector $\mathbf{n}_{i}$, oriented from grain $\beta$ to grain $\alpha$, and a tangential unit vector $\mathbf{t}_{i}$ are defined [13]. As long as the normal velocity $v_{n}\left(v_{n}=\left(\mathbf{v}_{i}^{\alpha}-\mathbf{v}_{i}^{\beta}\right) \cdot \mathbf{n}_{i}\right)$ remains positive, the normal force $f_{n}$ is identically zero. But when $v_{n}=0$, a non-negative (repulsive) normal force $f_{n}$ is mobilized at the contact node. These conditions define the velocity-Signorini complementary condition as shown in Fig. 4a $[9,16]$. On the other hand, by combining the equations of motion $\mathbf{P}_{\text {node }}^{\alpha}=\mathbf{M}^{\alpha} \mathbf{v}_{\text {node }}^{\alpha}$ and $\mathbf{P}_{\text {node }}^{\beta}=\mathbf{M}^{\beta} \mathbf{v}_{\text {node }}^{\beta}$ at the common node $i$, we get this linear relation:

$f_{n}=\frac{2}{\Delta t} \frac{m_{i}^{\alpha} m_{i}^{\beta}}{m_{i}^{\alpha}+m_{i}^{\beta}} v_{n}+k_{n}$,

where $m_{i}^{\alpha}$ and $m_{i}^{\beta}$ are the nodal masses of bodies of $\alpha$ and $\beta$, respectively, $\Delta t$ denotes the incremental time and $k_{n}$ is normal part of an offset force which depends on other contact forces exerted by the neighboring bodies of $\alpha$ and $\beta$. The normal forces at all contact nodes are obtained through an iterative process by intersecting the above linear relation with the Signorini graph, as shown in Fig. 4a.

In a similar vein, the Coulomb law of dry friction is a complementarity relation between the friction force $f_{t}$ and the tangential velocity $v_{t}\left(v_{t}=\left(\mathbf{v}_{i}^{\alpha}-\mathbf{v}_{i}^{\beta}\right) \cdot \mathbf{t}_{i}\right)$ at the contact

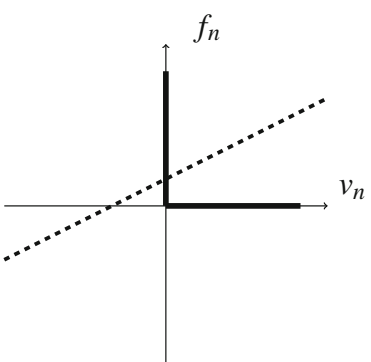

(a)

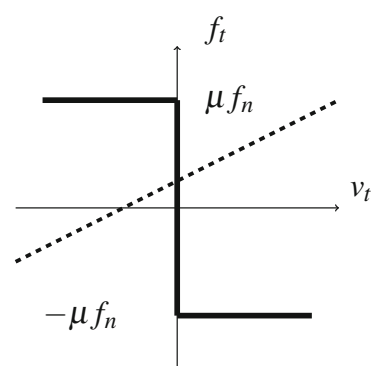

(b)
Fig. 4 Contact conditions: a velocity-Signorini complementarity condition as a graph relating the normal relative velocity $v_{n}$ and normal force $f_{n} ; \mathbf{b}$ coulomb friction law as a graph relating the tangential velocity $v_{t}$ and friction force $f_{t} ; \mu$ is the coefficient of friction. The dashed lines represent linear relations representing the equations of dynamics

node; see Fig. 4b. Like the Signorini graph, the Coulomb law is a complementarity relation in the sense that it can not be reduced to a single-valued function. The equations of motion at the common node $i$ yield this linear relation:

$f_{t}=\frac{2}{\Delta t} \frac{m_{i}^{\alpha} m_{i}^{\beta}}{m_{i}^{\alpha}+m_{i}^{\beta}} v_{t}+k_{t}$,

which is intersected with the Coulomb graph to calculate the friction force $f_{t}$ simultaneously at all contact nodes in the same iterative process used to calculate the normal forces. $k_{t}$ is tangentiel part of the offset force defined above. The convergence to the solution both for contact forces and internal stresses is smooth, and a high precision may be achieved through the convergence criterion. The compaction process of a packing of soft grains, discussed in the next section, provides an illustration of the application of this algorithm to granular materials.

\section{Axial deformation of a single grain}

Before analyzing the compaction of an assembly of soft grains, let us first consider the behavior of a single grain using the two methods described in Sect. 2. We performed BPM and MPM simulations of a soft grain of radius $R=5 \mathrm{~mm}$ and compressed between two rigid walls as shown in Fig. 5 . The bottom wall is fixed and the top wall moves downwards at a constant velocity of $0.2 \mathrm{~m} / \mathrm{s}$. In BPM simulations, the grain is composed of 1750 rigid frictionless particles. The diameters of the particles vary from $0.16 \mathrm{~mm}$ to $0.26 \mathrm{~mm}$. Their interactions are governed by Eq. (1) with $F_{0}=100$ $\mathrm{kN}, \gamma=7$ and a cut-off distance $\delta=a_{0}$. Note that $F_{0}$ is only a scaling factor in the sense that, according to Eq. (1) and since there is no intrinsic elastic force scale in the model, all forces should scale with $F_{0}$. Hence, its value has no effect on the behavior of the grains. 


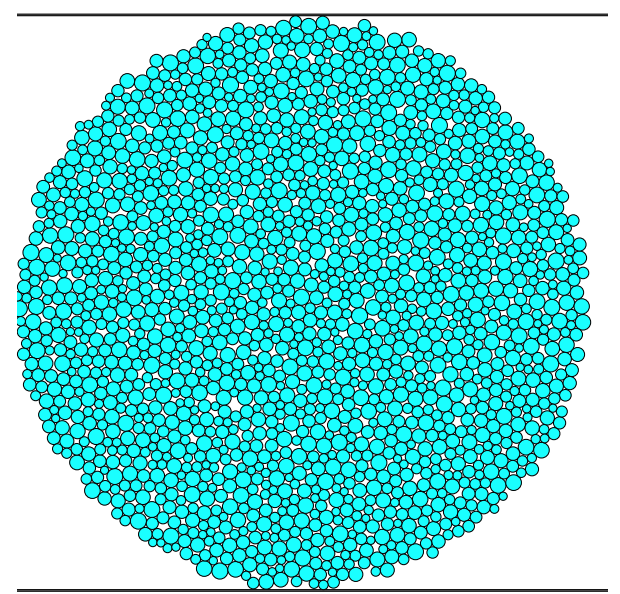

(a)

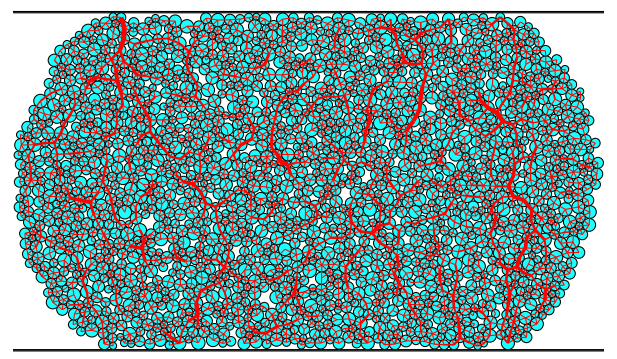

(c)

Fig. 5 Geometry of a soft grain by BPM (a) and MPM (b) discretization; deformed grain at cumulative vertical strain $\varepsilon=30 \%$ for the BPM (c) and for the MPM (d). In (c), the line thickness between particles is

Two-dimensional MPM simulations in plane strain conditions were performed. The computation domain was meshed with four-node quadrangular elements, and an initial distribution of four material points per element was used. Moreover, the material points were distributed so as to fit best to the initially circular shapes of the particles as shown in Fig. 5b. Young's modulus, Poisson's ratio and density of the particles were set to $E=10 \mathrm{MPa}, v=0.45$ and $\rho=990$ $\mathrm{kg} / \mathrm{m}^{3}$, respectively. We chose a high value of Poisson's ratio in order to get nearly constant volumes of the grains as in BPM grains. The choice of Young's modulus is indifferent since it acts only as a scale stress for the applied stress. In other words, it controls only the value of the applied stress for which a given level of deformation is reached. In the same way, the value of the density does not affect the behavior in the slow deformation regime. The spatial relative resolution is $\frac{\Delta r}{R}=0.029$, where $\Delta r$ is the mean distance between material points.

The deformed grains at vertical strain $\varepsilon=30 \%$ are shown in Fig. $5 \mathrm{c}, \mathrm{d}$ with force chains in the first case and von Mises stresses in the second case. The lateral curvatures are almost similar up to the surface roughness of the BPM grain. Close

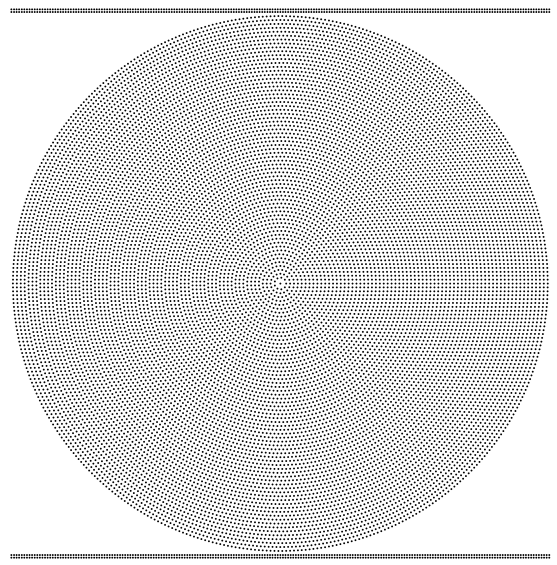

(b)

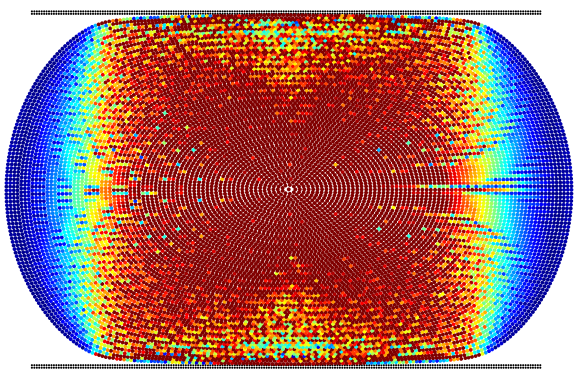

(d)

proportional to the normal repulsive force. In (d), the color code represents von Mises stresses of material points varying from lowest values in blue to largest values in red (color figure online)

inspection of the contact zone with the walls reveals a perfect contact line of the BPM grain with the top and bottom walls, reflecting its plastic behavior, whereas the MPM grain apparently touches the two walls only over a short segment at the center. But, in fact all material points belonging to the boundary elements between the particle and the bottom and top walls are in the contact line. This observed apparent gap is thus only a consequence of the background mesh element thickness.

Figure 6 shows the vertical stress $\sigma=\frac{F}{L}$, where $L$ is the actual largest section of the grain, as a function of axial strain. The MPM grain shows a linear elastic behavior at low deformation, as predicted by Hertz analysis for a disk [28]:

$$
F=\frac{\pi}{4} \frac{E}{1-v^{2}} R \varepsilon
$$

where $\varepsilon$ is the cumulative axial strain $(\varepsilon=\ln (1+d / R)$, $d$ being the displacement of the center of the grain). Deviation from the linear behavior is observed for $\varepsilon>0.05$. This range of the Hertz scaling (with an exponent $3 / 2$ in $3 D$ ) is generally used for molecular dynamics simulations of the 


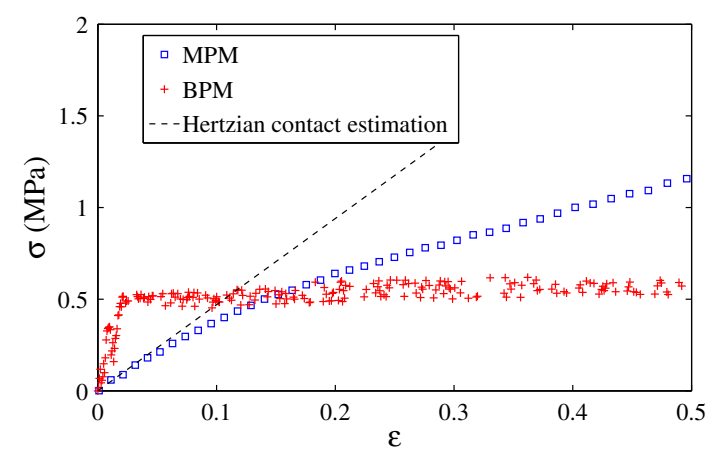

Fig. 6 Vertical stress as a function of cumulative axial strain for a single grain subjected to diametrical compression by means of BPM and MPM simulations

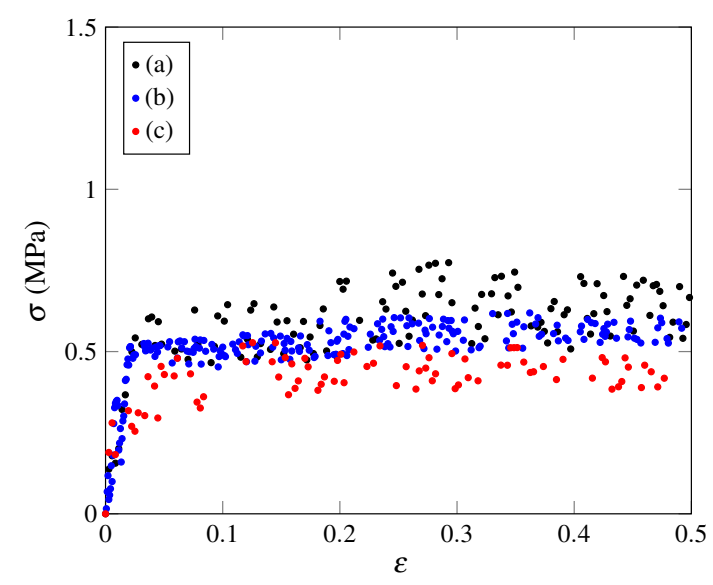

Fig. 7 Vertical stress as a function of cumulative axial strain for three BPM grains of the same size with a uniform size distribution of their primary particles but with different ranges of particles diameters: $a 2400$ particles with diameters in the range $[0.1,0.24] \mathrm{mm}, b 1750$ particles with diameters in the range $[0.16,0.26] \mathrm{mm}$ and $c 1300$ particles with diameters in the range $[0.22,0.28] \mathrm{mm}$

elastic behavior of granular materials composed of spherical particles $[22,39,40,44]$. The BPM grain is characterized by a linear response at small deformations of the order of $2 \%$ and a plastic behavior beyond. The plastic threshold stress is nearly constant and it reflects the internal characteristic stress $\sigma_{Y}=F_{0} /\left\langle a_{0}\right\rangle$. Its value in our simulations is approximately $0.5 \mathrm{MPa}$.

It is worth noting that the softness of the BPM grain depends on the size polydispersity of primary particles. It is expected that the plastic stress threshold will increase with size polydispersity. This is what we observe in Fig. 7 for three different size ploydispersities. In all the three examples, the primary particles have a uniform distribution of their volumes but the size ratio between the largest and smallest particles is varied. We see that grain "softness" slightly increases with size span.

\section{Compaction of a packing of soft grains}

In this section, we use both MPM and BPM simulations to investigate the compaction of a packing of soft grains. We consider the evolution of different packing parameters (packing fraction, connectivity) and their differences for the two methods. Since the accuracy and efficiency of the MPM algorithm has previously been shown through several contact problems [28], the comparison between the two methods allows us to demonstrate also the aptitude of the BPM to deal with soft-grain assemblies.

We consider a packing of 300 grains confined inside a rectangular box of width $L$ and of initial height $h_{0}$. The initial configuration is prepared by means of CD simulations. In both BPM and MPM simulations, the grain diameters have a uniform distribution by volume fractions in the range $[1.4,2.4] \mathrm{mm}$. Moreover, in each BPM grain, the particle diameters vary from 0.07 to $0.13 \mathrm{~mm}$ with a uniform distribution by particle volumes. The grains are subjected to vertical compaction by moving the top wall downwards at a constant velocity. We consider the same parameters as before for the two methods. Two simulations of compaction performed by the BPM and the MPM are analyzed below: (1) without friction and (2) with a coefficient of friction $\mu=0.5$ between the grains. The coefficient of friction between the grains and the walls is set to zero.

Figure 8(a-f) displays three snapshots of the compaction test with $\mu=0$ by BPM (a-c) and MPM (d-f) simulations. Since the compaction begins with a packing of unjammed particles, during the initial stage of compaction new contacts are formed between grains until they get jammed and the vertical stress begins to increase. For this point, the packing fraction increases by grain shape change, and at the end of the compaction nearly the whole space is filled by the grains. The shapes of the grains gradually change from circular to nearly polygonal as shown in Fig. 8g-h. Note that the 'gaps' observed in MPM simulations between grains reflect the meshing resolution, which may be increased for a finer discretization of the contact zone.

The evolution of the packing can be tracked through the mean coordination number $Z$ and the packing fraction $\Phi$ of the packing. Figure 9 shows $Z$ and $\Phi$ as a function of cumulative vertical strain $\varepsilon=\ln \left(1+\Delta h / h_{0}\right)$. The packing fraction $\Phi$ is a nearly linear function of $\varepsilon$. As $\Phi=1$ is approached, the behavior depends on that of individual grains. In MPM, the behavior is linear elastic and hence, the small remaining pores at high packing fraction can be filled by grain shape change only at very high stress level. In contrast, in BPM simulations the pores can be filled as a result of plastic deformation of the grains at much lower stress level. The process of pore filling is similar in MPM and BPM as long as the size of the inter-grain pores is above the size of primary particles. 


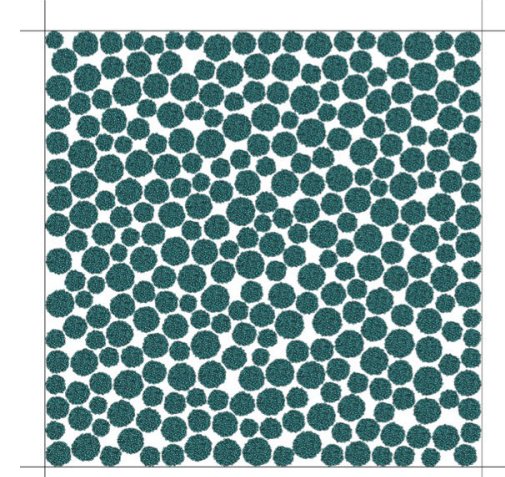

(a) $\varepsilon=0$ and $\Phi=0.65$

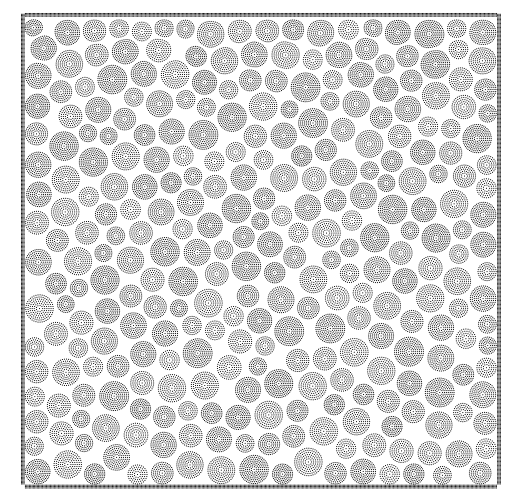

(d) $\varepsilon=0$ and $\Phi=0.65$

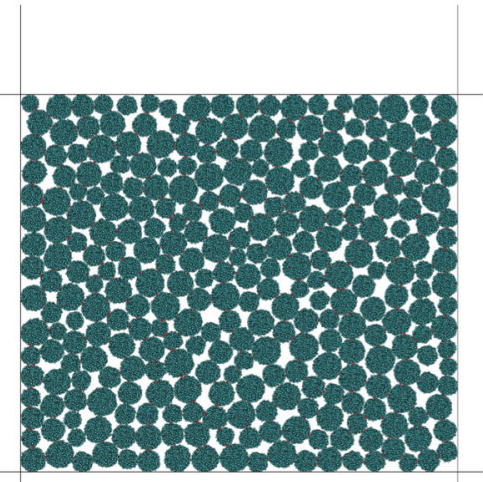

(b) $\varepsilon=0.17$ and $\Phi=0.8$

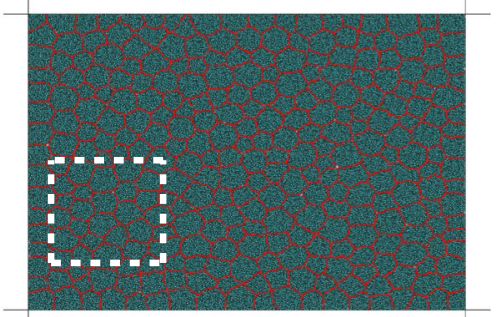

(c) $\varepsilon=0.45$ and $\Phi=0.99$

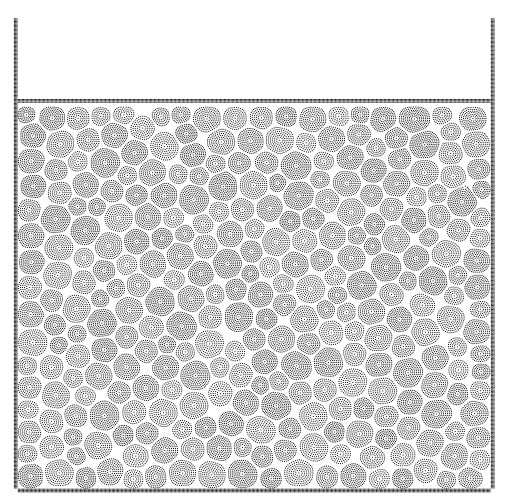

(e) $\varepsilon=0.17$ and $\Phi=0.8$

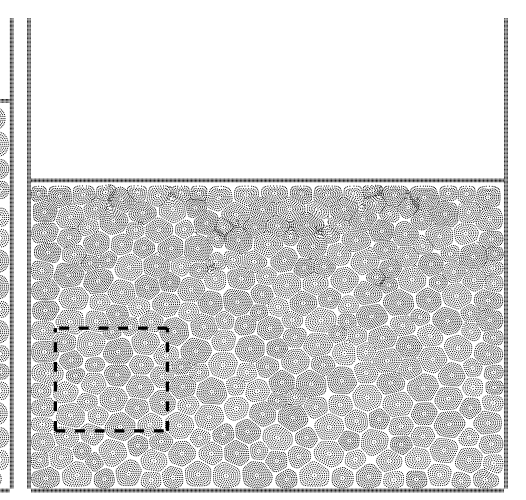

(f) $\varepsilon=0.45$ and $\Phi=0.99$

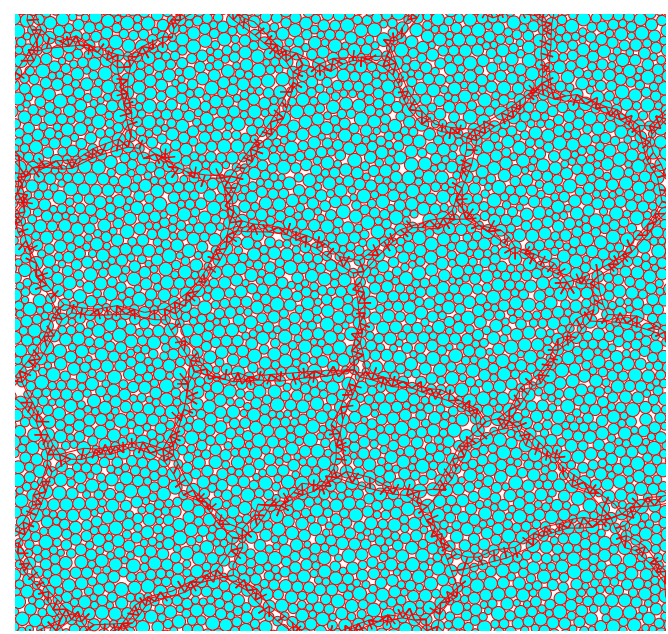

(g)

Fig. 8 Three snapshots of the compaction of a packing of soft grains by the BPM (a-c) and the MPM (d-f), and a zoom on the deformed packing by the BPM $(\mathbf{g})$ and by the MPM $(\mathbf{h})$. Filled circles are material

This limit occurs at a packing fraction of 0.93 in the BPM simulations.

The coordination number $Z$ increases with $\varepsilon$ in all cases. In the BPM simulations, we observe no difference between frictional and frictionless grains and the coordination number

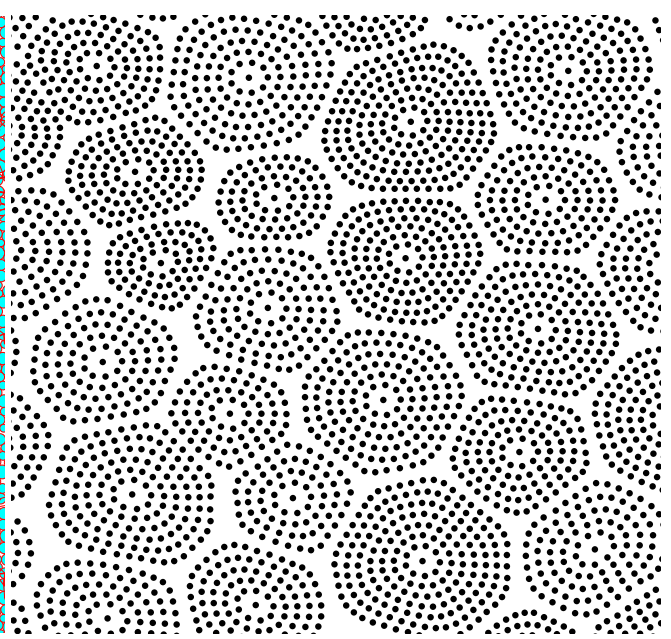

(h)

points in the MPM and the primary particles in the BPM. a $\varepsilon=0$ and $\Phi=0.65, \mathbf{b} \varepsilon=0.17$ and $\Phi=0.8, \mathbf{c} \varepsilon=0$ and $\Phi=0.65, \mathbf{d} \varepsilon=0.17$ and $\Phi=0.8, \mathbf{e} \varepsilon=0.45$ and $\Phi=0.99$

levels off after $\simeq 40 \%$ of deformation. In the MPM simulations, we observe higher values of $Z$ for frictionless grains. The jamming occurs at $\varepsilon \approx 0.08$ where $Z \approx 3$ and $\Phi \approx 0.75$. From this point, the vertical stress $\sigma$ begins to increase. However, the packing remains fragile until a packing fraction 

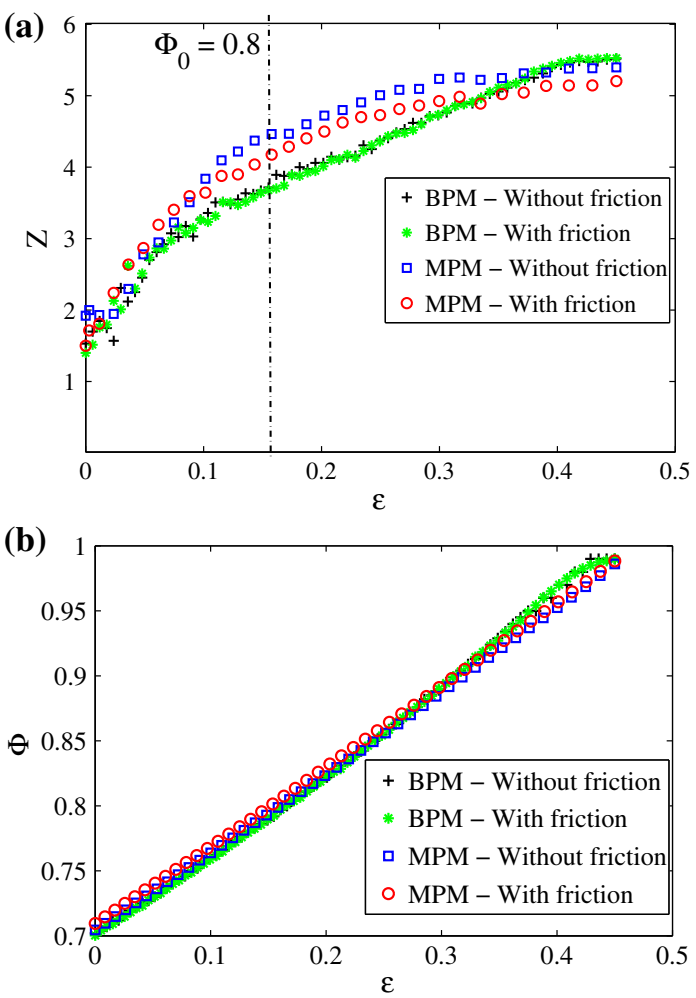

Fig. 9 Mean coordination number $Z$ (a) and packing fraction $\Phi(\mathbf{b})$ as a function of cumulative vertical strain $\varepsilon$ for frictionless and frictional particle packings by BPM and MPM simulations

$\Phi \approx 0.8$ is reached for a stress $\sigma_{0}$ that can be considered as consolidation stress. For this reason, we will use this stress to scale stresses during the subsequent deformations of the packing.

The fact that the inter-grain friction has no effect on $Z$ and $\Phi$ in BPM simulations is a simple consequence of the absence of friction between primary particles. Indeed, the friction force at the contact between two primary particles at the boundaries of two touching grains can not be mobilized since those particles can freely rotate without dissipation. In order to allow the friction to be effective at the inter-grain contacts, it is necessary to introduce rolling resistance between primary particles belonging to each grain. This resistance should be large enough to hinder relative rotations of the primary particles under the action of shear stresses [8,11,43]. Interestingly, as shown in Fig 9, in MPM simulations the packing fraction is the same in frictional and frictionless cases at all strain levels whereas $Z$ is higher in the frictionless case. This effect is observable from the jamming point at $\Phi \approx 0.75$.

Figure 10 shows the vertical stress $\sigma$, calculated from the forces acting on the bottom wall and normalized by the consolidation stress $\sigma_{0}$, as a function of the cumulative vertical strain $\varepsilon^{\prime}$ from the reference state defined by $\sigma=\sigma_{0}$. Here, we observe two very different behaviors in MPM and BPM sim-

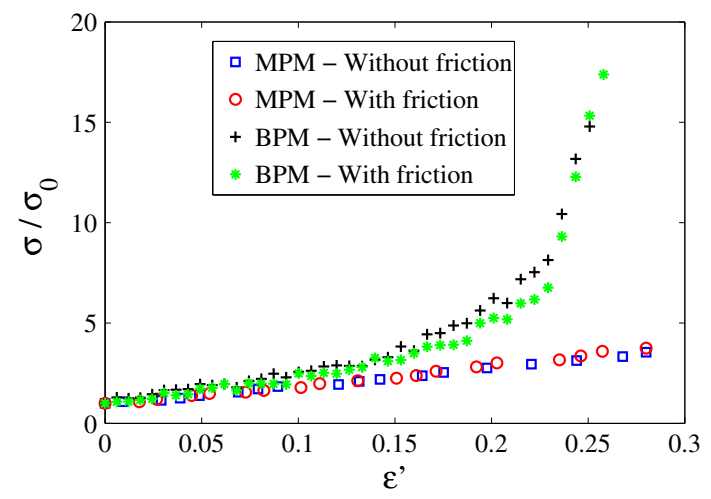

Fig. 10 Vertical stress $\sigma$ normalized by the consolidation stress as a function of cumulative vertical strain $\varepsilon^{\prime}$ from the reference state $\sigma_{0}$ for frictionless and frictional particle packings by BPM and MPM simulations

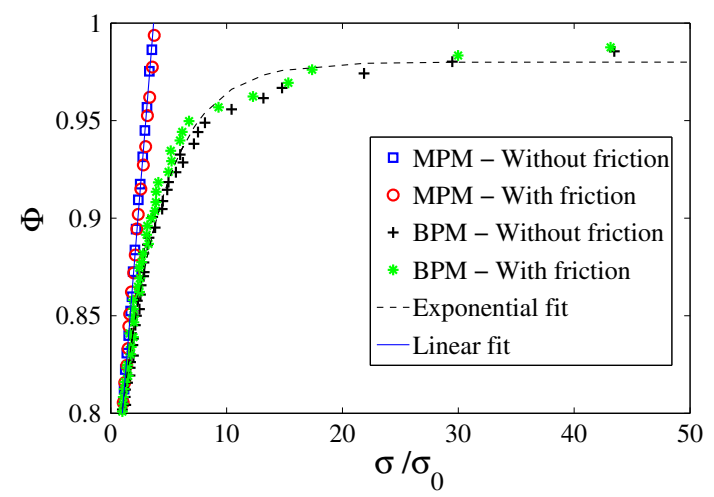

Fig. 11 Packing fraction as a function of normalized axial stress for frictionless and frictional particle packings subjected to uniaxial compression by BPM and MPM simulations. The dotted lines represent predictions by simple models of compaction introduced in this paper; see Eqs. (14) and (16)

ulations. In the MPM simulations, the behavior is a simple linear stress-strain relation, which clearly reflects the linear behavior of each grain, as observed in Fig. 6. In contrast, in the BPM simulations, the vertical stress increases at increasing rate with $\varepsilon^{\prime}$. This gradual increase of $\sigma$ is a consequence of the plastic behavior of BPM grains as seen in Fig. 6. Although each individual grain can reach its plastic stress threshold in the absence of other grains and geometrical constraints, the overall deformation of all grains of the packing depends on their mutual exclusions and the available pore volume. This is at the origin of stress divergence at large deformations. This is also what we observe when the packing fraction $\Phi$ is plotted as a function of $\sigma$; see Fig. 11. In MPM simulations, the packing fraction is a linear function of $\sigma$ until very high levels of packing fraction whereas in BPM simulations a nearly exponential increase of $\Phi$ is observed. It is also remarkable that inter-grain friction has almost no effect on the compaction process for both methods. 
The above observations about the evolution of the stress and packing fraction can be described on a quantitative basis by considering the deformation mechanisms for each method. In the BPM, since the grains are composed of rigid particles that can move and rearrange to fill the pore space between grains, it may be assumed that the variation $d \Phi$ of packing fraction is proportional to the available pore space and stress increment: $d \Phi \propto(1-\Phi) d \sigma$. This assumption readily leads to an exponential increase of $\Phi$ as a function of $\sigma$ :

$\Phi=1-\left(1-\Phi_{0}\right) e^{-\alpha\left(\frac{\sigma}{\sigma_{0}}-1\right)}$,

where $\alpha$ depends on the compressibility of the packing. Figure 11 shows that an exponential form fits quite well the $\mathrm{BPM}$ data up to a packing fraction $\simeq 0.98$ with $\alpha \simeq 0.27$. Beyond this limit, the pores between the grains are of nearly the same size as the pores inside the grains. For this reason, the evaluation of the packing fraction in this range is subject to fluctuations and the convention used for the determination of the interface between grains. In other words, the packing fraction is defined up to the porosity along all grain-grain interfaces inside the system.

On the other hand, in the MPM, since a continuum elastic model is used for the simulation of soft grains, the packing behaves like an elastic medium with an effective bulk modulus $K$ relating volume increment $d V$ to stress increment $d \sigma$ :

$K \frac{\mathrm{d} V}{V}=-\mathrm{d} \sigma$

Since the texture evolves mainly to the increase of intergrain interface, the strains may be assumed to be the same in grains and pores, so that the Voigt rule of mixtures can be applied [46]. Hence, the effective bulk modulus increases proportionally to the packing fraction: $K \propto \Phi K_{g}$, where $K_{g}$ is the grain bulk modulus (in $2 \mathrm{D}, K g=E / 2(1-v)$ ). Assuming further that the variation of the volume $V_{g}$ of grains is of second order compared to particle shape change, we obtain a linear relation between $\Phi$ and $\sigma$ :

$\Phi=\frac{\beta}{K_{g}}\left(\frac{\sigma}{\sigma_{0}}-1\right)+\Phi_{0}$.

This linear relation is consistent with our MPM simulations shown in Fig. 11 with a slope equal to $\beta / K_{g} \simeq 0.08$. Despite this difference, it is remarkable that the evolution of $\Phi$ as a function of $\sigma / \sigma_{0}$ is quite similar in the range $\Phi \in[0.8,0.87]$.

\section{Conclusion}

In this paper, we used two methods developed for the compaction of a packing of soft grains beyond the random close packing. The bonded particle model (BPM) is based on the representation of the grains as aggregates of rigid grains interacting via a hard-particle repulsive force at their contact points and an attraction force acting between particle centers up to a cut-off distance above one particle diameter. The material point method (MPM) is based on a discretization of the grains into moving material points. We implemented an implicit formulation of the MPM interfaced with the contact dynamics (CD) method for the treatment of frictional contacts. It was shown that, while MPM grains behave elastically by construction, the BPM grains have a perfectly plastic behavior.

The uniaxial compaction of a stack of soft grains was simulated using these two methods and the relationships between particle shape change and the evolution of packing fraction and coordination number were analyzed. The compaction with MPM grains is a linear process whereas the evolution in BPM simulations is nonlinear. By introducing two simple models, we showed that their difference can be explained in terms of their constitutive behaviors. The plastic behavior of BPM grains is a consequence of their discrete structure and inter-particle interactions. The plastic behavior can also be introduced in MPM simulations via the constitutive behavior of the grains. It is thus highly relevant to compare in the future the compaction of plastic MPM grains to that of BPM grains.

The BPM with deformable grains, as implemented for the first time in this paper, considerably extends the scope of the DEM in application to soft materials. The behavior of deformable grains can be calibrated or modified by using constitutive particles of different shapes and size distributions or by playing with the interactions between particles. For example, adding elastic interactions between primary particles leads to an elastic domain in the grain behavior. In the same way, the plastic behavior can be removed by adding rolling resistance and sliding friction between particles with very high values of friction coefficient and rolling resistance while keeping elastic interactions. Then, the behavior of a grain becomes purely elastic. If a force threshold is introduced on the attraction force and the friction coefficient is low, the grain behavior becomes brittle. To some respect, each grain can be made very similar to MPM grains with linear elastic behavior as long as the Young modulus is concerned. But the Poisson ratio of a BPM grain (with elastic interactions) is mainly controlled by the connectivity of the grains and it is of the order of $1 / 3$ in $2 \mathrm{D}$. These examples show that it is possible to use the BPM for different behaviors. But clearly the MPM is the best method for exact and straightforward implementation of the material behavior of grains.

In application to certain materials like clay, it is essential to account also for the cohesive forces between grains, which is straightforward to implement. In essence, the contacts 
between the particles represent nothing more than very hard repulsive potentials that occur between molecules. For this reason, the model can be applied to colloidal entities interacting by an effective attractive potential. The shear behavior of packings of such grains at high values of packing fraction can be simulated and analyzed by means of the BPM.

Acknowledgements Farhang Radjai would like to acknowledge the support of the ICoME2 Labex (ANR-11-LABX-0053) and the A*MIDEX projects (ANR-11-IDEX-0001-02) cofunded by the French program Investissements d'Avenir, managed by the ANR, the French National Research Agency.

\section{Compliance with ethical standards}

Conflict of interest Saeid Nezamabadi, Thanh Hai Nguyen, Jean-Yves Delenne and Farhang Radjai state that there are no conflicts of interest.

\section{References}

1. Agnolin, I., Roux, J.N.: Internal states of model isotropic granular packings. III. Elastic properties. Phys. Rev.E (Stat. Nonlinear Soft Matter Phys.) 76, 061304 (2007)

2. Antonyuk, S., Khanal, M., Tomas, J., Heinrich, S., Mörl, L.: Impact breakage of spherical granules: experimental study and dem simulations. Chem. Eng. Process. 45, 838-856 (2006)

3. Azéma, E., Radjai, F.: Stress-strain behavior and geometrical properties of packings of elongated particles. Phys. Rev. E 81, 051304 (2010)

4. Azéma, E., Radjai, F., Peyroux, R., Saussine, G.: Force transmission in a packing of pentagonal particles. Phys. Rev. E (Stat. Nonlinear Soft Matter Phys.) 76, 011301 (2007)

5. Bardenhagen, S., Brackbill, J., Sulsky, D.: The material-point method for granular materials. Comput. Methods Appl. Mech. Eng. 187, 529-541 (2000)

6. Bolton, M.D., N, Y., Cheng, P.: Micro- and macro-mechanical behaviour of dem crushable materials. Géotechnique 58, 471-480 (2008)

7. Bonnecaze, R., Cloitre, M.: Micromechanics of soft particle glasses. Adv. Polym. Sci. 236, 117-161 (2010)

8. Bratberg, I., Radjai, F., Hansen, A.: Dynamic rearrangements and packing regimes in randomly deposited two-dimensional granular beds. Phys. Rev. E 66(031), 303-1 (2002)

9. Brogliato, B.: Nonsmooth Mechanics. Springer, London (1999)

10. Cummins, S., Brackbill, J.: An implicit particle-in-cell method for granular materials. J. Comput. Phys. 180, 506-548 (2002)

11. Estrada, N., Taboada, A., Radjai, F.: Shear strength and force transmission in granular media with rolling resistance. Phys. Rev. E 78, 021301 (2008)

12. Guilkey, J., Weiss, J.: Implicit time integration for the material point method: quantitative and algorithmic comparisons with the finite element method. Int. J. Numer. Methods Eng. 57, 1323-1338 (2003)

13. Huang, P., Zhang, X., Ma, S., Huang, X.: Contact algorithms for the material point method in impact and penetration simulation. Int. J. Numer. Methods Eng. 85, 498-517 (2011)

14. Ioannidou, K., Pellenq, R., Gado, E.D.: Controlling local packing and growth in calcium-silicate-hydrate gels. Soft Matter 10, 1121 (2014)

15. Israelachvili, J.N.: Intermolecular and Surface Forces. Academic Press, London (1993)
16. Jean, M.: Frictional contact in rigid or deformable bodies: numerical simulation of geomaterials. In: Salvadurai, A., Boulon, J. (eds.) Mechanics of Geomaterial Interfaces, pp. 463-486. Elsevier Science Publisher, Amsterdam (1995)

17. Kabla, A.: Collective cell migration: leadership, invasion and segregation. J. R. Soc. Interface 9, 3268-3278 (2012)

18. Lepesanta, P., Bohera, C., Berthierb, Y., Rézai-Ariaa, F.: A phenomenological model of the third body particles circulation in a high temperature contact. Wear 298-299, 66-79 (2013)

19. Liu, L., Kafui, K., Thornton, C.: Impact breakage of spherical, cuboidal and cylindrical agglomerates. Powder Technol. 199, 189196 (2010)

20. Lorenzo, G., Zartizky, N., Califano, A.: Rheological analysis of emulsion-filled gels based on high acyl gellan gum. Food Hydrocoll. 30, 672-680 (2013)

21. Ma, G., Zhou, W., Chang, X.L.: Modeling the particle breakage of rockfill materials with the cohesive crack model. Comput. Geotech. 61, 132-143 (2014)

22. Makse, H.A., Johnson, D., Schwartz, L.: Packing of compressible granular materials. Phys. Rev. Lett. 84, 4160-4163 (2000)

23. Menut, P., Seiffert, S., Sprakelae, J., Weitz, D.: Does size matter? elasticity of compressed suspensions of colloidal- and granularscale microgels. Soft Matter 8, 156-164 (2012)

24. Moreau, J.: Some numerical methods in multibody dynamics: application to granular materials. Eur. J. Mech. A. Solids 13, 93114 (1994)

25. Moreau, J.J.: Evolution problem associated with a moving convex set in a hilbert space. J. Differ. Equ. 26, 347-374 (1977)

26. Moreau, J.J.: Bounded variation in time. In: Panagiotopoulos, P., Strang, G. (eds.) Topics in Nonsmooth Mechanics, pp. 1-74. Bikhäuser, Basel (1988)

27. Moreno, R., Ghadiri, M., Antony, S.: Effect of the impact angle on the breakage of agglomerates: a numerical study using dem. Powder Technol. 130, 132-137 (2003)

28. Nezamabadi, S., Radjai, F., Averseng, J., Delenne, J.Y.: Implicit frictional-contact model for soft particle systems. J. Mech. Phys. Solids 83, 72-87 (2015)

29. Nguyen, D.H., Azéma, E., Sornay, P., Radjai, F.: Bonded-cell model for particle fracture. Phys. Rev. E 91, 022203 (2015)

30. Peyneau, P.E., Roux, J.N.: Frictionless bead packs have macroscopic friction, but no dilatancy. Phys. Rev. E 78, 011307 (2008)

31. Quezada, J.C., Breul, P., Saussine, G., Radjai, F.: Stability, deformation, and variability of granular fills composed of polyhedral particles. Phys. Rev. E 86, 031308 (2012)

32. Radjai, F., Dubois, F.: Discrete Numerical Modelling of Granular Materials. Wiley-ISTE, Berlin (2011)

33. Radjai, F., Jean, M., Moreau, J., Roux, S.: Force distributions in dense two-dimensional granular systems. Phys. Rev. Lett. 77, 274 (1996)

34. Radjai, F., Richefeu, V.: Contact dynamics as a nonsmooth discrete element method. Mech. Mater. 41, 6715-728 (2009)

35. Radjai, F., Roux, S.: Turbulentlike fluctuations in quasistatic flow of granular media. Phys. Rev. Lett. 89, 064302 (2002)

36. Radjai, F., Wolf, D.E., Jean, M., Moreau, J.: Bimodal character of stress transmission in granular packings. Phys. Rev. Lett. 80, 61-64 (1998)

37. Saint-Cyr, B., Delenne, J.Y., Voivret, C., Radjai, F., Sornay, P.: Rheology of granular materials composed of nonconvex particles. Phys. Rev. E 84, 041302 (2011)

38. Sator, N., Mechkov, S., Sausset, F.: Generic behaviours in impact fragmentation. Europhys. Lett. 81, 44002 (2008)

39. Silbert, L.E., Grest, G.S., Brewster, R., Levine, A.J.: Rheology and contact lifetimes in dense granular flow. Phys. Rev. Lett. 99, 068002 (2007) 
40. Singh, A., Magnanimo, V., Saitoh, K., Luding, S.: The role of gravity or pressure and contact stiffness in granular rheology. New J. Phys. 17, 043028 (2015)

41. Staron, L., Vilotte, J.P., Radjai, F.: Preavalanche instabilities in a granular pile. Phys. Rev. Lett. 89, 204302 (2002)

42. Taboada, A., Chang, K.J., Radjai, F., Bouchette, F.: Rheology, force transmission, and shear instabilities in frictional granular media from biaxial numerical test using the contact dynamics method. J. Geophys. Res. 110, 1-24 (2005)

43. Taboada, A., Estrada, N., Radjaï, F.: Additive decomposition of shear strength in cohesive granular media from grain-scale interactions. Phys. Rev. Lett. 97, 098302 (2006)

44. Thornton, C.: Force transmission in granular media. KONA Powder Part. 15, 81-90 (1997)
45. Thornton, C., Yin, K.K., Adams, M.J.: Numerical simulation of the impact fracture and fragmentation of agglomerates. J. Phys. D Appl. Phys. 29, 424-435 (1996)

46. Torquato, S.: Random Heterogeneous Materials-Microstructure and Macroscopic Properties. Springer, New York (2002)

47. Wang, J., Yan, H.: On the role of particle breakage in the shear failure behavior of granular soils by dem. Int. J. Numer. Anal. Meth. Geomech. 37, 832-854 (2013)

48. Wittel, F., Carmona, H., Kun, F., Herrmann, H.: Mechanisms in impact fragmentation. Int. J. Fract. 154, 105-117 (2008) 